Pacific Journal of Mathematics

ON A PROBLEM OF O. TAUSSKY 


\section{ON A PROBLEM OF O. TAUSSKY}

\section{Bernard W. LEVINGER AND Richard S. VARGA}

Recently, 0 . Taussky raised the following question. Given a nonnegative $n \times n$ matrix $A=\left(a_{i}, j\right)$, let $\AA_{A}$ be the set of all $n \times n$ complex matrices defined by

$$
\stackrel{\circ}{A}_{A} \equiv\left\{B=\left(b_{i, j}\right)|| b_{i, j} \mid=a_{i, j} \text { for all } 1 \leqq i, j \leqq n\right\} .
$$

Then, defining the spectrum $S(\mathfrak{M})$ of an arbitrary set $\mathfrak{M}$ of $n \times n$ matrices $B$ as

$$
S(\mathfrak{M}) \equiv\{\sigma \mid \operatorname{det}(\sigma I-B)=0 \text { for some } B \in \mathfrak{M}\},
$$

what can be said in particular about $S\left(\AA_{A}\right)$ ? It is not difficult to see that $S\left(\AA_{A}\right)$ consists of possibly one disk and a series of annular regions concentric about the origin, but our main result is a precise characterization of $S\left(\Omega_{A}\right)$ in terms of the minimal Gerschgorin sets for $A$.

Introduction. We shall distinguish between two cases. If there is a diagonal matrix $D=\operatorname{diag}\left(x_{1}, \cdots, x_{n}\right)$ with $\boldsymbol{x} \geqq \mathbf{0}$ and $\boldsymbol{x} \neq \mathbf{0}$ such that $A D$ is diagonally dominant, then $A$ is called essentially diagonally dominant. In this case, the set $S\left(\AA_{A}\right)$ is just the minimal Gerschgorin set $G\left(\Omega_{A}\right)$ of [6], rotated about the origin (Theorem 1 and Corollary 2). Determining $S\left(\AA_{A}\right)$ in this case is quite easy, since it suffices to determine those points of the boundary of $G\left(\Omega_{A}\right)$ which lie on the positive real axis (Theorem 2). This is discussed in $\S 2$.

In the general case when $A$ is not essentially diagonally dominant, we must use permutations and intersections (Theorem 3) to fully describe $S\left(\AA_{A}\right)$, in the spirit of [3]. These results are described in $\S 3$. Also in this section is a generalization (Theorems 3 and 4) of a recent interesting result by Camion and Hoffman [1]. Our proof of this generalization differs from that of [1].

Finally, in $\S 4$ we give several examples to illustrate the various possibilities for $S\left(\AA_{A}\right)$.

Before leaving this section, we point out that the question posed by 0 . Taussky [5, p. 129] has an immediate answer in terms of the results of [3]. In [3], the authors completely characterized the spectrum $S\left(\Omega_{0}\right)$ of a related set $\Omega_{0}$ of matrices, where $C=\left(c_{i, j}\right)$ was an arbitrary $n \times n$ complex matrix and

$$
\left.\Omega_{o} \equiv\left\{B=\left(b_{i, j}\right)|| b_{i, j}\right)=\left|c_{i, j}\right| \text { and } b_{i, j}=c_{i, j} \text { for all } 1 \leqq i, j \leqq n\right\} .
$$

Clearly, $\Omega_{A} \subset \AA_{A}$. On the other hand, if $D(\theta)$ represents an $n \times n$ diagonal matrix all of whose diagonal entries have modulus unity: 
$d_{j, j}=\exp \left(i \theta_{j}\right), 1 \leqq j \leqq n$, then $A D(\theta) \subset \stackrel{\Omega}{\Delta}_{\Delta}$ and $\stackrel{\Omega}{\Delta}_{\Delta}=\mathbf{U}_{\theta} \Omega_{\Delta D(\theta)}$, where the union is over all possible choices of $D(\theta)$. Thus,

$$
S\left(\AA_{A}\right)=\bigcup_{\forall} S\left(\Omega_{\Delta D(\theta)}\right) .
$$

While this answers the question posed, it neither gives an insight into the nature of $S\left(\stackrel{\Omega}{A}_{A}\right)$, nor allows $S\left(\AA_{A}\right)$ to be effectively calculated. We shall show that in fact $S\left(\Omega_{A}\right)$ is more easily determined than $S\left(\Omega_{A}\right)$.

2. The essentially diagonally dominant case. Let $A=\left(a_{i, j}\right)$ be given $n \times n$ nonnegative matrix. In order to develop the material of this section, we recall some definitions and results concerning the minimal Gerschgorin set $G\left(\Omega_{A}\right)$ associated with $A$. In $[3,6]$, a continuous real-valued function $\nu(\sigma)$, defined for all complex numbers $\sigma$, was characterized by

$$
\nu(\sigma) \equiv \inf _{\boldsymbol{u}>0} \max _{i}\left\{\frac{1}{u_{i}}\left[\sum_{j \neq i} a_{i, j} u_{j}-\left|\sigma-a_{i, i}\right| u_{i}\right]\right\} .
$$

Using the Perron-Frobenius theory of nonnegative matrices [7, 2.4 and $\S 8.2$, it can be shown that there exists a nonnegative vector $\boldsymbol{x} \neq \boldsymbol{0}$ such that

$$
-\left|\sigma-a_{i, i}\right| x_{i}+\sum_{j \neq i} a_{i, j} x_{j}=\nu(\sigma) x_{i}, \quad 1 \leqq i \leqq n .
$$

From $\nu(\sigma), G\left(\Omega_{4}\right)$ is defined by

$$
G\left(\Omega_{\Delta}\right)=\{\sigma \mid \nu(\sigma) \geqq 0\} .
$$

In view of $\left(2.1^{\prime}\right)$ and (2.2), a complex number $\sigma$ is contained in $G\left(\Omega_{A}\right)$ if and only if there is a nonnegative vector $\boldsymbol{x} \neq \mathbf{0}$ such that

$$
\left|\sigma-a_{i, i}\right| x_{i} \leqq \sum_{\jmath \neq i} a_{i, j} x_{j}, \quad 1 \leqq i \leqq n .
$$

The set $G\left(\Omega_{\boldsymbol{A}}\right)$ is a closed bounded set, and its boundary, denoted by $\partial G\left(\Omega_{4}\right)$, satisfies,

$$
\partial G\left(\Omega_{A}\right) \subset S\left(\Omega_{A}\right) \subset G\left(\Omega_{A}\right) .
$$

We first prove a result concerning $G\left(\Omega_{A}\right)$ which will have later applications.

Lemma 1. If, for $z_{0}>0, z_{0} e^{i \theta} \in G\left(\Omega_{\Delta}\right)$ for all real $\theta$, then all $z$ with $|z| \leqq z_{0}$ are in $G\left(\Omega_{A}\right)$, and $z=0$ is an interior point of $G\left(\Omega_{A}\right)$.

Proof. This is a simple application of (2.3). By assumption, 
$-z_{0} \in G\left(\Omega_{4}\right)$. Since $z_{0}>0$ and $a_{i, i} \geqq 0,1 \leqq i \leqq n$, then

$$
\left|-z_{0}-a_{i, i}\right|=z_{0}+a_{i, i} \text {. }
$$

Thus, for any $z$ with $|z| \leqq z_{0}$,

$$
\left|z-a_{i, i}\right| \leqq|z|+a_{i, i} \leqq z_{0}+a_{i, i},
$$

and (2.3) holds for $z$ with the same vector $\boldsymbol{x} \geqq 0$ which satisfies (2.3) for $-z_{0}$, which completes the proof.

We next introduce the notion of rotating a given point set $P$ about the origin. Let

$$
\operatorname{rot} P \equiv\left\{\sigma \mid \sigma e^{i \theta} \in P \text { for some real } \theta\right\} .
$$

With this notation, we have

LemMa 2. $\operatorname{rot} S\left(\stackrel{\Omega}{A}_{A}\right)=S\left(\stackrel{\Omega}{A}_{A}\right)$.

Proof. It is clear that $S\left(\AA_{A}\right) \subset \operatorname{rot} S\left(\AA_{A}\right)$. If $\sigma \in \operatorname{rot} S\left(\AA_{A}\right)$, then $\sigma e^{i \theta}$ is an eigenvalue of some $B$ in $\AA_{A}$ and thus $\sigma$ is an eigenvalue of $e^{-i \theta} B$. But $e^{-i \theta} B \in \stackrel{\circ}{\Lambda}_{A}$ and hence $\sigma \in S\left(\stackrel{\circ}{A}_{A}\right)$, which completes the proof. This elementary result already establishes that the spectrum $S\left(\Omega_{\Delta}\right)$ can be described as the union of a family of circles concentric about the origin.

LEMMA 3. If $\sigma \in S\left(\AA_{A}\right)$, then $|\sigma| \in G\left(\Omega_{A}\right)$.

Proof. For any $\sigma \in S\left(\AA_{\Delta}\right)$, there is a matrix $B=\left(b_{i, j}\right)$ in $\AA_{\Delta}$ and a vector $\boldsymbol{y} \neq \mathbf{0}$ such that $\dot{B y}=\sigma \boldsymbol{y}$. Equivalently, we have

$$
\left(\sigma-b_{i, i}\right) y_{i}=\sum_{j \neq i} b_{i, j} y_{j}, \quad 1 \leqq i \leqq n
$$

If we take absolute values in (2.6) and note that

$$
\left|\sigma-b_{i, i}\right| \geqq|| \sigma|-| b_{i, i}||=|| \sigma\left|-a_{i, i}\right|,
$$

we obtain

$$
|| \sigma\left|-a_{i, i}\right|\left|y_{i}\right| \leqq\left|\sigma-b_{i, i}\right|\left|y_{i}\right|=\left|\sum_{j \neq i} b_{i, j} y_{j}\right| \leqq \sum_{j \neq i} a_{i, j}\left|y_{i}\right|,
$$

so that $|\boldsymbol{\sigma}|$ satisfies (2.3) with the nonnegative vector $\boldsymbol{x}=|\boldsymbol{y}|$, which completes the proof.

From the definition (2.5), it follows that, if $P$ and $R$ are any sets with $P \subset R$, then $\operatorname{rot} P \subset \operatorname{rot} R$. Thus, (2.4) and Lemma 3 combine to give

COROLLARY 1. $\operatorname{rot} \partial G\left(\Omega_{4}\right) \subset S\left(\AA_{\Delta}\right) \subset \operatorname{rot} G\left(\Omega_{4}\right)$. 
We now study the case for which the inclusions of Corollary 1 become equalities.

THEOREM 1. Let $A$ be a nonnegative $n \times n$ matrix. Then, $\operatorname{rot} \partial G\left(\Omega_{A}\right)=S\left(\AA_{\Delta}\right)=\operatorname{rot} G\left(\Omega_{\Delta}\right)$ if and only if $z=0$ is not an interior point of $G\left(\Omega_{A}\right)$.

Proof. First, assume that $z=0 \notin \operatorname{int} G\left(\Omega_{A}\right)$, and let $\sigma$ be an arbitrary nonzero point of $\operatorname{rot} G\left(\Omega_{A}\right)$, so that $\sigma e^{i \theta_{0}} \in G\left(\Omega_{A}\right)$ for some real $\theta_{0}$. The circle $|z|=|\sigma|$ cannot lie entirely in $G\left(\Omega_{A}\right)$. For otherwise, by Lemma 1 , the entire disk $|z| \leqq|\sigma|$ would be contained in $G\left(\Omega_{A}\right)$ and $z=0$ would be an interior point of $G\left(\Omega_{A}\right)$. Thus, the circle $|z|=|\sigma|$ necessarily intersects the boundary $\partial G\left(\Omega_{A}\right)$, and there exists a real $\theta_{1}$ such that $\sigma e^{i \theta_{1}} \in \partial G\left(\Omega_{A}\right)$. It follows that $\sigma \in \operatorname{rot} \partial G\left(\Omega_{A}\right)$, and thus from Corollary $1, \sigma$ is also a point of $S\left(\AA_{A}\right)$. To complete this part of the proof, we need only examine the point $z=0$. Clearly, the statement that $0 \notin \operatorname{int} G\left(\Omega_{A}\right)$ is equivalent to the statement that either $0 \in G^{\prime}\left(\Omega_{A}\right)$, the complement of $G\left(\Omega_{A}\right)$, or $0 \in \partial G\left(\Omega_{A}\right)$. Thus, if $0 \in \operatorname{rot} G\left(\Omega_{A}\right)$, i.e., $0 \in G\left(\Omega_{A}\right)$, then the previous remark shows that $0 \in \partial G\left(\Omega_{A}\right)$, which completes the proof of the first part. Now, assume that $\operatorname{rot} \partial G\left(\Omega_{\mathbf{A}}\right)=S\left(\Omega_{\mathbf{A}}\right)=\operatorname{rot} G\left(\Omega_{\mathbf{A}}\right)$, and call this common set of points $H$. If $0 \in H$, then $0 \in \partial G\left(\Omega_{A}\right)$, and hence $0 \notin \operatorname{int} G\left(\Omega_{\Delta}\right)$. If $0 \notin H$, then $0 \notin G\left(\Omega_{A}\right)$, which implies that $0 \in G^{\prime}\left(\Omega_{A}\right)$, and again $0 \notin \operatorname{int} G\left(\Omega_{A}\right)$, which completes the proof.

The statement $z=0 \notin \operatorname{int} G\left(\Omega_{4}\right)$ can be seen to be equivalent to $\nu(0) \leqq 0$, and this has an interesting connection with diagonally dominant matrices, i.e., $n \times n$ matrices $B=\left(b_{i, j}\right)$ satisfying

$$
\left|b_{i, i}\right| \geqq \sum_{j \neq i}\left|b_{i, j}\right|, \quad 1 \leqq i \leqq n .
$$

Obviously, if $\nu(0) \leqq 0$, then from $\left(2.1^{\prime}\right)$, there is a nonnegative vector $\boldsymbol{y} \neq \mathbf{0}$ such that

$$
a_{i, i} y_{i} \geqq \sum_{j \neq i} a_{i, j} y_{j}, \quad 1 \leqq i \leqq n .
$$

Thus, if $D$ is the diagonal matrix $D \equiv \operatorname{diag}\left(y_{1}, \cdots, y_{n}\right)$, then (2.9) asserts that the product $A D$ is diagonally dominant. Conversely, if $D=\operatorname{diag}\left(y_{1}, \cdots, y_{n}\right)$ where $\boldsymbol{y} \geqq \mathbf{0}$ and $\boldsymbol{y} \neq \mathbf{0}$ and $A D$ is diagonally dominant, then it follows from (2.3) that $\nu(0) \leqq 0$.

The statement that $\nu(0) \leqq 0$ can also be coupled with results of Ostrowski [4] on $H$-matrices, which are defined as follows. Let $B=\left(b_{i, j}\right)$ be an arbitrary $n \times n$ complex matrix, and associate with $B$ the new matrix $C=\left(c_{i, j}\right)$, where $c_{i, j}=-\left|b_{i, j}\right|, i \neq j$, and

$$
c_{i, i}=\left|b_{i, i}\right|, \quad 1 \leqq i \leqq n .
$$


Then, $B$ is an $H$-matrix if and only if all the principal minors of $C$ are nonnegative. [That is, the matrix $C$ is a possibly degenerate $M$-matrix.] In [4], it is shown that $B$ is an $H$-matrix if and only if there exists a diagonal matrix $D=\operatorname{diag}\left(y_{1}, \cdots, y_{n}\right)$ with $\boldsymbol{y} \geqq \mathbf{0}, \boldsymbol{y} \neq \mathbf{0}$, such that $B D$ is diagonally dominant. Thus we have

CoROLlary 2. Let $A$ be a nonnegative $n \times n$ matrix. Then, $\operatorname{rot} \partial G\left(\Omega_{\Delta}\right)=S\left(\Omega_{\Delta}\right)=\operatorname{rot} G\left(\Omega_{\Delta}\right)$ if and only if $A$ is an $H$-matrix.

Summarizing, we have shown that the sets $\operatorname{rot} \partial G\left(\Omega_{A}\right), S\left(\AA_{A}\right)$, and $\operatorname{rot} G\left(\Omega_{A}\right)$ are equal in the case that $A$ is an $H$-matrix, and this might logically be called the essentially diagonally dominant case, the title of this section.

We have already shown that $S\left(\AA_{A}\right)$ is a collection of annuli and disks concentric about the origin. It is now logical to ask how the radii of these regions can be determined. For convenience, we will assume that $A$ is irreducible (cf. [7, p. 20]). The reducible case requires only minor modifications.

We consider the function $\nu(t)$ along the nonnegative real axis $t \geqq 0$. Let $\left\{t_{i}\right\}_{i=1}^{m}$ define the finite sequence of points $t_{1}>t_{2}>\cdots$ $>t_{m}>0$, such that $\nu\left(t_{i}\right)=0$ and $\nu\left(t_{i}+\varepsilon\right) \cdot \nu\left(t_{i}-\varepsilon\right)<0$ for all sufficiently small $\varepsilon>0$. Then, these points $t_{i}$ indicate strong sign changes in $\nu(t)$. In [6], it was shown that the spectral radius of $A$,

$$
\rho(A) \equiv \max _{i}\left\{\left|\lambda_{i}\right| \mid \operatorname{det}\left(\lambda_{i} I-A\right)=0\right\},
$$

is such a point, and since it was further shown that $\nu(\rho(A)+\delta)<0$ for all $\delta>0$, it is evidently the largest such point, i.e., $t_{1}=\rho(A)$ and $m \geqq 1$. We define $t_{m+1}=0$, and now show that the points $t_{i}$ divide the nonnegative real axis into intervals in which $\nu(t) \geqq 0$.

Lemma 4. For $t \geqq 0, \nu(t) \geqq 0$ if and only if $t_{2 i} \leqq t \leqq t_{2 i-1}$ for some $i$ with $1 \leqq i \leqq[(m+1) / 2]$.

Proof. Since $\nu(t)$ is continuous for $t \geqq 0$, it suffices to show that there is no $\mu>0$, corresponding to a degenerate change of signs, with $\nu(\mu)=0$ such that $\nu(\mu-\varepsilon)<0$ and $\nu(\mu+\varepsilon)<0$ for all sufficiently small $\varepsilon>0$. This assertion is basically a consequence of the assumption that $A$ is irreducible. For, if such a $\mu>0$ exists, then $\mu \in \partial G\left(\Omega_{A}\right)$. Moreover, since $\left|t e^{i \theta}-a_{i, i}\right|>\left|t-a_{i, i}\right|$ for any $t>0$ and any real $\theta$ with $0<|\theta| \leqq \pi$, it follows from (2.1) that $\nu\left(t e^{i \theta}\right)<\nu(t)$ and hence that $\nu(z)<0$ for all complex $z \neq \mu$ in a neighborhood of $\mu$. Thus, $\mu$ is an isolated point of $G\left(\Omega_{A}\right)$. As such, it follows [6] that $\mu$ is necessarily a diagonal entry of $A$, i.e., $\mu=a_{j, j}$ for some $j$. But, since 
$A$ is irreducible, it is known [6] that $\nu\left(a_{k, k}\right)>0$ for every $1 \leqq k \leqq n$. This contradiction establishes the desired result.

THEOREm 2. Let $A$ be a nonnegative irreducible $n \times n$ matrix, and let $t_{1}>t_{2}>\cdots>t_{m}>0$ be positive real numbers such that $\boldsymbol{\nu}\left(t_{i}\right)=0$ and $\boldsymbol{\nu}\left(t_{i}+\varepsilon\right) \cdot \boldsymbol{\nu}\left(t_{i}-\varepsilon\right)<0$ for all sufficiently small $\varepsilon>0$. If $m>1$ and $z$ is any complex number with $|z| \geqq t_{2[m / 2]}$, then $z \in S\left(\Omega_{A}\right)$ if and only if $t_{2 i} \leqq|z| \leqq t_{2 i-1}$ for some $i$ with $1 \leqq i \leqq[m / 2]$.

Proof. If $z_{0}$ is any complex number with $\left|z_{0}\right| \geqq t_{2[m / 2]}$ and $t_{2 i} \leqq\left|z_{0}\right| \leqq t_{2 i-1}$ for some $1 \leqq i \leqq[m / 2]$, then from Lemma $4, \nu\left(\left|z_{0}\right|\right) \geqq 0$. Also, from Lemma 4 it follows that $\nu(|z|)<0$ for any $|z|$ with $t_{2 i+1}<|\boldsymbol{z}|<t_{2 i}$. Thus, all points in the disk $|\boldsymbol{z}| \leqq\left|z_{0}\right|$ are not points of $G\left(\AA_{\Delta}\right)$, and we deduce from Lemma 1 that $\left|z_{0}\right| e^{i \theta} \in \partial G\left(\Omega_{A}\right)$ for some real $\theta$. Thus, $z_{0} \in \operatorname{rot} \partial G\left(\Omega_{A}\right)$, and thus from Corollary $1, z_{0} \in S\left(\AA_{A}\right)$, which proves one part of this result. Conversely, for any $z_{0} \in S\left(\Omega_{\Delta}\right)$ with $\left|z_{0}\right| \geqq t_{2[m / 2]}, \nu\left(\left|z_{0}\right|\right) \geqq 0$ from Lemma 3 . Then from Lemma 4, it follows that $t_{2 i} \leqq\left|z_{0}\right| \leqq t_{2 i-1}$ for some $i$ with $1 \leqq i \leqq[m / 2]$, which completes the proof.

Using the results of [6], it is now simple to determine the exact number of eigenvalues of any matrix $B \in \check{\Omega}_{\boldsymbol{A}}$ which lie in each of the outer annuli: $t_{2 i} \leqq|\boldsymbol{z}| \leqq t_{2 i-1}$ for $1 \leqq i \leqq[m / 2]$.

COROLlary 3. Let $A$ be a nonnegative irreducible $n \times n$ matrix with $m>1$. Then, for any $B \in \stackrel{\Omega}{A}_{A}, B$ has $p_{i}$ eigenvalues in the annulus $t_{2 i} \leqq|z| \leqq t_{2 i-1}, 1 \leqq i \leqq[m / 2]$, if and only if $A$ has $p_{i}$ diagonal entries in this annulus.

Proof. By a familiar continuity argument, going back to Gerschgorin, each connected component of $S\left(\AA_{A}\right)$ contains the same number of eigenvalues for each $B \in \check{\Omega}_{\boldsymbol{A}}$, and hence, the same number as $A$. But from [6], $A$ has $p_{i}$ eigenvalues in this annulus if and only if $A$ has $p_{i}$ diagonal entries in this annulus, which completes the proof.

As final remarks in this section, we mention that Theorem 2 precisely gives $S\left(\AA_{A}\right)$ and the radii of its associated concentric annuli in the case that $m$ (the number of strong sign changes in $\nu(t)$ for $t \geqq 0$ ) is even. In this regard, it is interesting to point out that the geometrical result of Theorem 1 and Corollary 2 is basically contained in Theorem 2, since it can be obtained by applying Theorem 2 to a family of nonnegative irreducible matrices $A(\varepsilon), \varepsilon \geqq 0$, where $A(\varepsilon) \rightarrow A$ 
as $\varepsilon \downarrow 0$, for which $m$ is again even for each $A(\varepsilon)$ for all sufficiently small $\varepsilon>0$. We also mention that computing the points $t_{i}$ or Theorem 2 , whether $m$ is even or odd, is not difficult because of the inclusion relationships of $(2.1)$.

In the case that $m=2 l+1$ is odd, Theorem 2 gives no information about the final disk $0 \leqq|z| \leqq t_{2 l+1}$, and different techniques are necessary to decide which points of this disk are points of $S\left(\AA_{4}\right)$. This will be discussed in $\S 3$.

3. $\nu(0)>0$. If $z=0$ is an interior point of $G\left(\Omega_{A}\right)$, i.e., $\nu(0)>0$, we can still give a precise characterization of $S\left(\AA_{\Delta}\right)$ using the methods of [3], but these results are considerably more complicated than those given in $\S 2$. We shall show by means of examples in $\S 4$ that these complications cannot, unfortunately, be avoided.

We first give a more or less well known result.

LEMMA 5. Let $0 \leqq \alpha_{1} \leqq \alpha_{2} \leqq \cdots \leqq \alpha_{n}$ be nonnegative real numbers, and $\rho$ an arbitrary complex number. Then, there exist real numbers $\theta_{1}, \cdots, \theta_{n}$ such that $\rho=\sum_{j=1}^{n} \alpha_{j} e^{i \theta}$ if and only if

$$
\sum_{j=1}^{n} \alpha_{j} \geqq|\rho| \geqq \alpha_{n}-\sum_{j=1}^{n-1} \alpha_{j}
$$

Proof. This lemma is precisely Lemma 1 of [1] applied to the $n+1$ nonnegative numbers $\alpha_{1}, \cdots, \alpha_{n},|\rho|$. However, for completeness, we give a proof by induction.

Only the fact that (3.1) implies the existence of the $\theta_{j}$ is nontrivial. For $n=2,\left|\alpha_{2}+\alpha_{1} e^{i \theta}\right|=\sqrt{\alpha_{2}^{2}+2 \alpha_{1} \alpha_{2} \cos \theta+\alpha_{1}^{2}}$ which varies continuously from $\alpha_{2}+\alpha_{1}$ to $\alpha_{2}-\alpha_{1}$ as $\theta$ varies from 0 to $\pi$.

For $n+1$, we distinguish two cases. Consider first the case where $|\boldsymbol{\rho}| \geqq\left|\alpha_{n+1}-\sum_{i=1}^{n} \alpha_{i}\right|$. Then, as in the previous case for $n=2$, for some $\theta$ we can write $|\rho|=\left|\alpha_{n+1}+e^{i \theta} \sum_{i=1}^{n} \alpha_{i}\right|$. Otherwise, if $|\boldsymbol{\rho}|<\left|\alpha_{n+1}-\sum_{i=1}^{n} \alpha_{i}\right|$, then from (3.1) we deduce that $|\rho|<\sum_{i=1}^{n} \alpha_{i}-\alpha_{n+1}$, which gives us the inequalities

$$
\alpha_{n}-\sum_{i=1}^{n-1} \alpha_{i} \leqq \alpha_{n} \leqq|\rho|+\alpha_{n+1} \leqq \sum_{i=1}^{n} \alpha_{i} .
$$

Thus, from the inductive hypothesis, $\alpha_{n+1}+|\rho|$, and hence also $\rho$, have the representations of the desired form.

With this, we now characterize $S\left(\AA_{\mathbf{S}}\right)$ by a set of linear inequalities. 
LEMMA 6. Let $\sigma$ be an arbitrary complex number. Then $\sigma \in S\left(\AA_{\mathbf{4}}\right)$ if and only if there exists a nonnegative vector $\boldsymbol{x} \neq \mathbf{0}$ such that

$$
\sum_{j=1}^{n} a_{i, j} x_{j} \geqq|\sigma| x_{i} \geqq a_{i, k} x_{k}-\sum_{j \neq k} a_{i, j} x_{j}
$$

for each $i$ and $k$ with $1 \leqq i, k \leqq n$.

Proof. If $\sigma \in S\left(\AA_{\Delta}\right)$, there exists a matrix $B \in \AA_{\Delta}$ and a vector $\boldsymbol{z} \neq \mathbf{0}$ with $B z=\sigma z$. Taking absolute values and setting $\left|z_{j}\right|=x_{j}$, we obtain for the $i$-th component

$$
\sum_{j=1}^{n} a_{i, j} x_{j} \geqq\left|\sum_{j=1}^{n} b_{i, j} x_{j}\right|=|\sigma| x_{i} \geqq a_{i, k} x_{k}-\sum_{j \neq k} a_{i, j} x_{j},
$$

for each $1 \leqq k \leqq n$, which establishes the first part of this theorem. Conversely, if (3.2) is satisfied by a nonnegative vector $\boldsymbol{x} \neq \mathbf{0}$ for each $i$ and $k, 1 \leqq i, k \leqq n$, we can repeatedly apply Lemma 5 to find real constants $\theta_{k, j}$ such that $\sigma x_{k}=\sum_{j=1}^{n} a_{k}, e^{i \theta_{k}, j} x_{j}$ for $1 \leqq k \leqq n$, so that $\sigma \in S\left(\Omega_{4}^{\circ}\right)$, which completes the proof.

We now remark that the inequalities of (3.2) are equivalent to the following set of $n^{2}$ linear inequalities

$$
\begin{aligned}
\sum_{j \neq i}(-1)^{\delta_{j, k}} a_{i, j} x_{j}+(-1)^{\delta_{i, k}}|| \sigma\left|+(-1)^{\delta_{i, k}} a_{i, i}\right| & x_{i} \geqq 0, \\
1 & \leqq i, k \leqq n,
\end{aligned}
$$

where $\delta_{i, k}$ is the Kronecker delta function. For $k \neq i$, the second inequality of (3.2) is identical with (3.3). For $k=i$, (3.2) yields

$$
\sum_{j \neq i} a_{i, j} x_{j} \geqq\left(|\sigma|-a_{i, i}\right) x_{i} \geqq-\sum_{j \neq i} a_{i, j} x_{j},
$$

which is equivalent to

$$
\sum_{j \neq i} a_{i, j} x_{j}-|| \sigma\left|-a_{i, i}\right| x_{i} \geqq 0 .
$$

In order to develop the material of this section, we recall some definitions and results [3] concerning the minimal Gerschgorin set $G^{\varphi}\left(\Omega_{\sigma}\right)$ associated with a matrix $C$ relative to the permutation $\varphi$. Let $C=\left(c_{i, j}\right)$ be an arbitrary $n \times n$ complex matrix, and let $\varphi$ be any permutation of the first $n$ positive integers. If $\sigma$ is any complex number, we can define a continuous real valued function $\nu_{\varphi, \sigma}(\sigma)$ by

$$
\begin{aligned}
\nu_{\varphi, \sigma}(\sigma)= & \inf _{u>0} \max _{i}\left\{\frac { 1 } { u _ { \varphi ( i ) } } \left[\sum_{j \neq i}(-1)^{\delta_{j, \varphi(i)}}\left|c_{i, j}\right| u_{j}\right.\right. \\
& \left.\left.+(-1)^{\delta_{i, \varphi(i)}}\left|\sigma-c_{i, i}\right| u_{i}\right]\right\} .
\end{aligned}
$$


The minimal Gerschgorin set $G^{\varphi}\left(\Omega_{\sigma}\right)$ is given as in (2.2) by

$$
G^{\varphi}\left(\Omega_{\sigma}\right)=\left\{\sigma \mid \nu_{\varphi, \sigma}(\sigma) \geqq 0\right\} .
$$

Equivalently, $\sigma \in G^{\varphi}\left(\Omega_{\sigma}\right)$ if and only if there exists a nonnegative vector $\boldsymbol{x} \neq \mathbf{0}$ such that

$$
\sum_{j \neq i}(-1)^{\delta j, \varphi(i)}\left|c_{i, j}\right| x_{j}+(-1)^{\delta_{i, \varphi(i)}}\left|\sigma-c_{i, i}\right| x_{i} \geqq 0, \quad 1 \leqq i \leqq n .
$$

In order to couple the inequalities (3.3) to those of $(3.6)$, let $A^{\varphi}=\left(a_{i, j}^{\varphi}\right)$ be an $n \times n$ matrix derived from $A$ as follows:

$$
a_{i, j}^{\varphi}=\left\{\begin{array}{ll}
a_{i, j}, & j \neq i \\
(-1)^{1+\delta_{i, \varphi(i)}} a_{i, i}, & j=i
\end{array}\right\}, \quad 1 \leqq i, j \leqq n .
$$

It is clear from Lemma 6 and the definition of $A_{\varphi}$ that $\sigma \in S\left(\AA_{A}\right)$ implies that $|\sigma| \in G^{\varphi}\left(\Omega_{\left.\mathbf{A}^{p}\right)}\right.$ for each permutation $\varphi$. Note that this result generalizes Lemma 3 of $\S 2$ to arbitrary permutation. Hence, it follows that $|\sigma| \subset \bigcap_{\varphi} G^{\varphi}\left(\Omega_{\mathbb{A}^{\varphi}}\right)$, so that

$$
S\left(\AA_{A}\right) \subset \operatorname{rot}\left(\bigcap_{\varphi} G^{\varphi}\left(\Omega_{\Delta^{\varphi}}\right)\right) .
$$

We now show that equality is valid in (3.8).

TheOREm 3. Let $A=\left(a_{i, j}\right)$ be a nonnggative $n \times n$ matrix. Then,

$$
S\left(\AA_{\Delta}\right)=\operatorname{rot}\left(\bigcap_{\varphi} G^{\varphi}\left(\Omega_{A^{\varphi}}\right)\right) .
$$

Proof. From (3.8), it suffices to show that $|\sigma| \in \bigcap_{\varphi} G^{\varphi}\left(\Omega_{\mathbb{A}^{\varphi}}\right)$ implies that $|\sigma| \in S\left(\AA_{A}\right)$. To prove this, we define the sets $M_{i, k}(|\sigma|)$ from (3.3) by

$$
\begin{aligned}
M_{i, k}(|\boldsymbol{\sigma}|)= & \left\{\boldsymbol{x} \geqq \mathbf{0} \mid \sum_{j=1}^{n} x_{j}=1 ; \sum_{j \neq i}(-1)^{\delta_{j, k}} a_{i, j} x_{j}\right. \\
& \left.+(-1)^{\delta_{i, k}}|| \boldsymbol{\sigma}\left|+(-1)^{\delta_{i, k}} a_{i, i}\right| x_{i} \geqq 0\right\} .
\end{aligned}
$$

By (3.3), $|\sigma| \in S\left(\AA_{A}\right)$ is equivalent to the existence of a vector $\boldsymbol{x}$ with

$$
\boldsymbol{x} \in \bigcap_{1 \leqq i, k \leqq n} M_{i, k}(|\sigma|),
$$

and thus we must prove that $\bigcap_{1 \leqq i, k \leq n} M_{i, k}(|\sigma|)$ is nonempty. We shall show that the hypothesis, $|\sigma| \in \bigcap_{\varphi} G^{\varphi}\left(\Omega_{A^{\varphi}}\right)$, implies that any $n$ of the sets $M_{i, k}(|\sigma|)$ have a nonempty intersection. Then, the conclusion will follow from Helly's Theorem [2, p. 33], which states that if $K$ is a family of at least $n$ convex sets in Euclidean $(n-1)$-space, 
$R^{n-1}$, such that every subclass containing $n$ members has a common point in $R^{n-1}$, there is a point common to all members of $K$. Since the $M_{i, k}(|\sigma|)$ are convex and of dimension at most $(n-1)$, this implies our theorem.

It remains to show that any collection $\left\{M_{i_{y}, k_{j}}(|\sigma|)\right\}_{j=1}^{n}$ has a nonempty intersection. This is always true if the second subscript $k_{j}$ fails to take on the integer value $k_{0}, 1 \leqq k_{0} \leqq n$. For, if $\boldsymbol{y}$ is the vector with components $y_{k_{0}}=1, y_{j}=0$ for $j \neq k_{0}$, we see that (3.3) is satisfied and thus $\boldsymbol{y} \in \bigcap_{j=1}^{n} M_{i_{y}, k_{j}}(|\sigma|)$. By (3.6) and (3.7), the condition $|\sigma| \in G^{\varphi}\left(\Omega_{A^{\varphi}}\right)$ is equivalent to the assertion that $\bigcap_{\varphi} M_{i, \varphi(i)}(|\sigma|)$ is nonempty. Thus, $|\sigma| \in \bigcap_{\varphi} G^{\varphi}\left(\Omega_{\Delta^{\varphi}}\right)$ implies that $\bigcap_{j=1}^{n} M_{i_{\jmath}, k_{j}}(|\sigma|)$ is nonempty whenever $k_{j}=\varphi\left(i_{j}\right)$ for some permutation $\varphi$. Finally, consider a collection $\left\{M_{j(k), k}\right\}_{k=1}^{n}$ where $j(k)$ is not one-to-one. In this case, there is evidently a repeated first index, and for convenience, we assume that $1=j(1)=j(2)=\cdots=j(r), r \geqq 2$. Then let $\boldsymbol{y}$ be any nonnegative vector with $y_{1}+y_{2}=1, y_{j}=0$ for $2<j \leqq n$. For such vectors, it follows from (3.9) that

$$
\boldsymbol{y} \in M_{1,1} \text { if and only if } a_{1,2} y_{2}-|| \sigma\left|-a_{1,1}\right| y_{1} \geqq 0,
$$

$$
\boldsymbol{y} \in M_{1,2} \text { if and only if }-a_{1,2} y_{2}+|| \sigma\left|+a_{1,1}\right| y_{1} \geqq 0 \text {, }
$$

$$
\boldsymbol{y} \in M_{j(k), k}, k>2 \text { if and only if } a_{j(k), 1} y_{1}+a_{j(k), 2} y_{2} \geqq 0 \text {. }
$$

Clearly, from $\left(3.10^{\prime \prime}\right)$ all such vectors $\boldsymbol{y}$ are in $\bigcap_{k>2} M_{j(k), k}$. If $a_{1,2}>0$, then the vector $\boldsymbol{y}$ with $y_{2}=\left(|| \sigma\left|-a_{1,1}\right| y_{1}\right) / a_{1,2}$ is in $M_{1,1} \cap M_{1,2}$, and if $a_{1,2}=0$, then the vector $\boldsymbol{y}$ with $y_{2}=1 y_{1}=0$ is in $M_{1,1} \cap M_{1,2}$. Thus, $\bigcap_{k=1}^{n} M_{j(k), k}$ is nonempty, and we conclude that any collection of $n$ sets $M_{i, j}$ has a nonempty intersection, which completes the proof.

We can further show that, if $\sigma \notin S\left(\AA_{A}\right)$, then as in [1] there is a unique permutation $\varphi$ such that $|\sigma| \notin G^{\varphi}\left(\Omega_{\mathbf{A}^{\varphi}}\right)$. This will permit us to show that at most $(n+1)$ permutations are necessary to characterize $S\left(\AA_{A}\right)$ in Theorem 3 .

THEOREM 4. If $\sigma \notin S\left(\AA_{A}\right)$, then there exists a unique permutation $\varphi$ such that $|\sigma| \notin G^{\varphi}\left(\Omega_{\mathbf{A}^{\varphi}}\right)$.

Proof. If $\sigma \notin S\left(\AA_{\Delta}\right)$, then, by Theorem 3 , there is at least one permutation $\varphi$ with $|\sigma| \notin G^{\varphi}\left(\Omega_{A^{\varphi}}\right)$. Thus, if $|\sigma| \notin G^{\psi}\left(\Omega_{A^{\psi}} \psi\right.$, we must show that $\psi=\varphi$, i.e., $\psi(i)=\varphi(i)$ for $1 \leqq i \leqq n$.

To prove this, we introduce the sets

$$
\begin{aligned}
N_{i, k}= & \left\{\boldsymbol{x} \geqq 0 \mid \sum_{j=1}^{n} x_{j}=1 ; \sum_{j \neq i}(-1)^{\delta_{j k} k} a_{i, j} x_{j}\right. \\
& \left.+(-1)^{\delta_{i, k}}|| \sigma\left|+(-1)^{\delta_{i, k}} a_{i, i}\right| x_{i}<0\right\},
\end{aligned}
$$


with $1 \leqq i, k \leqq n$. Clearly, $N_{i, k}$ is the complement of $M_{i, k}(|\sigma|)$ relative to the $(n-1)$-simplex $S \equiv\left\{\boldsymbol{x} \geqq \mathbf{0} \mid \sum_{j=1}^{n} x_{j}=1\right\}$. It is also clear that $N_{i, k}$ is empty if and only if $a_{i, k}=0$ when $i \neq k$, and ||$\sigma\left|-a_{i, i}\right|=0$ whem $i=k$, and $N_{i, k}$ does not intersect the face of the simplex $S$ defined by $x_{k}=0$. Further, it is readily verified that $N_{i, k} \cap N_{i, k^{\prime}}$ is empty if $k \neq k^{\prime}$.

If $|\sigma| \notin G^{\varphi}\left(\Omega_{\mathbb{A}^{\varphi}}\right)$, it follows from (3.6) and (3.7) that $S=\bigcap_{i=1}^{n} N_{i, \varphi(i)}$. On the other hand, $|\sigma| \notin G^{\varphi}\left(\Omega_{\Delta^{\varphi}}\right)$ implies from (3.5) that $\nu_{\varphi, \mathbf{A}^{\varphi}}(|\sigma|)<0$, and hence, from the definition of (3.4), there must exist (by continuity) a positive vector $\boldsymbol{u}>\mathbf{0}$ with $\boldsymbol{u} \in N_{i, \varphi(i)}$ for all $1 \leqq i \leqq n$, i.e., if $\boldsymbol{u}$ is normalized, then $\boldsymbol{u} \in \bigcap_{i=1}^{n} N_{i, \varphi(i)}$. Similarly, $|\sigma| \notin G^{\psi}\left(\Omega_{\boldsymbol{A}} \psi\right)$ implies that $S=\bigcap_{i=1}^{n} N_{i, \psi(i)}$.

Now, let $I=\{j \mid \psi(j)=\varphi(j), 1 \leqq j \leqq n\}$. Assuming that $\psi \neq \varphi$, then $I$ is a proper subset of the first $n$ positive integers. From the vector $\boldsymbol{u}>\mathbf{0}$ above, form the vector $\boldsymbol{v} \in S$ as follows: $v_{\varphi(j)}=0, j \in I$; $v_{\varphi(j)}=u_{\varphi(j)}, u_{\varphi(j)} /\left(\sum_{j \notin I} u_{\varphi(j)}\right), j \notin I$. Since $u \in N_{i, \varphi(i)}$ for all $1 \leqq i \leqq n$, it is easy to verify that $v \in N_{i, \varphi(i)}$ for any $i \notin I$, and thus $\boldsymbol{v} \in \boldsymbol{\bigcap}_{i \notin I} N_{i, \varphi(i)}$. Furthermore, $\boldsymbol{v} \in \mathbf{\bigcup}_{i \notin I} N_{i, \psi(i)}$ since the union of the $N_{i, \psi(j)}$ covers the simplex $S$, and $N_{j, \psi(j)}$ does not intersect the face $v_{\varphi(j)}=0$ for $j \in I$. Thus, there is a $k \notin I$ such that $v \in N_{k, \psi(k)} \cap N_{k, \varphi(k)}$. But since $N_{i, k} \cap N_{i, k^{\prime}}$ is empty if $k \neq k^{\prime}$, then it follows that $\psi(k)=\varphi(k)$, i.e., $k \in I$, which contradicts the assumption that $I$ is a proper subset of the first $n$ positive integers. Hence, $\varphi(i)=\psi(i)$ for all $1 \leqq i \leqq n$, which completes the proof.

We remark that the special case $\sigma=0$ of Theorems 3 and 4 corresponds to the main results of [1].

Letting $R^{\prime}$ denote the complement of any set $R$ in the complex plane, then Theorem 4 implies:

Corollary 4. If $K$ is an open connected component of $\left(S\left(\AA_{A}\right)\right)^{\prime}$, the complement of $S\left(\Omega_{\mathbf{A}}\right)$, then there is a unique permutation $\psi$ for which $K \subset\left(G^{\psi}\left(\Omega_{A} \psi\right)\right)^{\prime}$.

Proof. Since $\bigcap_{\varphi} G^{\varphi}\left(\Omega_{\mathbb{A}^{\varphi}} \subset S\left(\AA_{\Delta}\right)\right.$ by Theorem 3, then obviously $\left(S\left(\Omega_{A}\right)\right)^{\prime} \subset\left(\bigcap_{\varphi} G^{\varphi}\left(\Omega_{A^{\varphi}}\right)\right)^{\prime}=\bigcup_{\varphi}\left(G^{\varphi}\left(\Omega_{A^{\varphi}}\right)\right)^{\prime}$. Next, we remark that if $|\sigma|$ were replaced by $\sigma$ in the definition of $N_{i, k}$ in (3.11), all subsequent arguments remain valid. In particular, from the proof of Theorem 4, it follows that the $\left(G^{\varphi}\left(\Omega_{A^{\varphi}}\right)\right)^{\prime}$ are nonintersecting open sets. Thus, the open connected component $K$ can be in only one set $\left(G^{\psi}\left(\Omega_{\Delta} \psi\right)\right)^{\prime}$, which completes the proof. We remark that in general $K \neq\left(G_{\psi}^{\psi}\left(\Omega_{A} \psi\right)\right)^{\prime}$ because of the rotational invariance of any connected component of $\left(S\left(\Omega_{A}\right)\right)^{\prime}$.

We now consider the closed connected components of $S\left(\AA_{A}\right)$. 
THEOREM 5. Every connected component of $S\left(\AA_{\mathbf{A}}\right)$ contains the same number of eigenvalues for each matrix $B$ in $\dot{\Omega}_{\Delta}$.

Proof. This is basically a continuity argument. For, given any matrix $B \in \stackrel{\Omega}{A}_{\Delta}$, we can construct a matrix $B(t) \in \stackrel{\Omega}{A}_{\mathbf{A}}$ whose entries are continuous functions of $t, 0 \leqq t \leqq 1$, such that $B(0)=A$ and $B(1)=B$. Since the eigenvalues of $B(t)$ then vary continuously with $t$, each matrix $B \in \check{\Omega}_{A}$ must have the same number of eigenvalues as $A$ in each connected component of $S\left(\Omega_{\Delta}\right)$, which completes the proof.

Theorem 3 states that $S\left(\Omega_{4}\right)$ can be determined from the $n$ ! sets $G^{\varphi}\left(\Omega_{A^{\varphi}}\right)$. The next result shows that at most $(n+1)$ permutations are necessary for the determination of $S\left(\dot{\Omega}_{4}\right)$.

THEOREM 6. There exist permutations $\varphi_{1}, \varphi_{2}, \cdots, \varphi_{r}$ with $r \leqq n+1$ such that $S\left(\AA_{A}\right)=\operatorname{rot}\left(\bigcap_{i=1}^{r} G^{\varphi}\left(\Omega_{A} \varphi_{i}\right)\right)$.

Proof. Since the matrix $A$ has $n$ eigenvalues, then $S\left(\AA_{A}\right)$ can have at most $n$ closed connected components by Theorem 6 . Because each closed connected component of $S\left(\AA_{\Delta}\right)$ is either a (possibly degenerate) disk or an annulus centered at the origin, then it is clear that the complement of $S\left(\dot{\Omega}_{4}^{\circ}\right)$ consists of at most $(n+1)$ similar regions. By Corollary 3 , exactly one permutation corresponds to each open connected component of $\left(S\left(\Omega_{A}^{\circ}\right)\right)$, and thus at most $(n+1)$ permutations are necessary to describe $S\left(\dot{\Omega}_{A}\right)$.

We remark that, since $\left(S\left(\AA_{A}\right)\right)^{\prime}$ always contains the unbounded connected component $\{z|| z \mid>\rho(A)\}$, the identity permutation must always occur as one of the $r$ permutations of Theorem 6 . This follows from the fact [3] that $G^{\varphi}\left(\Omega_{A^{\varphi}}\right)$ is a bounded set only for the identity permutation. Of course, if $A$ is essentially diagonally dominant, then $r=1$ from Theorem 1. We now remark that the results of Theorem 2 and Corollary 3 can be used to obtain an improved upper bound for $r$. For, if $t_{m}$ is, as in Theorem 2, the smallest positive number such that $\nu\left(t_{m}\right)=0$, then by Corollary 3 , the number of eigenvalues $\sigma$ for each $B \in \Omega_{A}^{\circ}$ with $|\sigma| \geqq t_{m}$ is equal to the number, $k$, of diagonal entries $a_{i, i}$ of $A$ with $a_{i, i} \geqq t_{m}$, and clearly $k \geqq[m / 2]$. Thus, by the same argument as above,

$$
r \leqq n+1-k
$$

In $\S 4$, we give an example of a $3 \times 3$ matrix for which 3 permutations are required to determine $S\left(\Omega_{4}^{\circ}\right)$. In general, examples can similarly be given where $n$ permutations are required for the $n \times n$ case, and we conjecture that the result of Theorem 6 is valid with 
$n+1$ reduced to $n$.

To actually calculate $S\left(\Omega_{A}\right)$ in the general case, it is necessary from Corollary 4 to work with the complements of the sets $G^{\varphi}\left(\Omega_{\Lambda^{\varphi}}\right)$, i.e., to determine those intervals of the positive real axis $(t \geqq 0)$ for which $\nu_{\varphi, 4^{\varphi}}(t)<0$ for some permutation $\varphi$. However, it is in general not easy to determine a priori which $r(\leqq n+1)$ of the $n$ ! permutations suffice to characterize $S\left(\AA_{4}\right)$ in Theorem 6. For this reason, the analogue of Theorem 2 which could be stated for the general case seems computationally unattractive.

4. Examples. To illustrate the results of $\S 2$, consider the following diagonally dominant matrix $A$ :

$$
A=\left[\begin{array}{lll}
1 & 1 / 2 & 0 \\
1 / 2 & 3 & 1 / 2 \\
0 & 1 / 2 & 5
\end{array}\right] .
$$

For this matrix, the minimal Gerschgorin set $G\left(\Omega_{A}\right)$ is given by

$$
G\left(\Omega_{A}\right)=\{z: 4|z-1| \cdot|z-3| \cdot|z-5| \leqq|z-5|+|z-1|\} .
$$

From this, it can be verified that the intervals of the nonnegative real axis for which $\nu(t) \geqq 0$ are given by

$$
0.88 \leqq t \leqq 1.14 ; 2.75 \leqq t \leqq 3.25 ; 4.86 \leqq t \leqq 5.12 \text {. }
$$

From Theorem 2, $S\left(\Omega_{A}\right)$ then consists of three concentric annuli, and from Corollary 3 , each $B \in \Omega_{A}^{\circ}$ has exactly one eigenvalue in each annulus.

To illustrate the results of $\S 3$, consider the matrix $A(\varepsilon)$ where

$$
A(\varepsilon)=\left[\begin{array}{lll}
\varepsilon & 1 & 0 \\
0 & \varepsilon & 1 \\
1 & 2 & \varepsilon
\end{array}\right],
$$

and $\varepsilon \geqq 0$. Note that $A(0)$ is the companion matrix for the polynomial $x^{3}-2 x-1$. It is not difficult to show that at most three permutations $\mathbf{s}^{1}, \varphi_{1}=I, \varphi_{2}=(23), \varphi_{3}=(123)$, are necessary to describe $S\left({\left.\stackrel{\circ}{A_{(\varepsilon)}}\right)}_{\text {, }}\right.$, i.e., $G^{\varphi}\left(\Omega_{\Delta(\varepsilon)^{\varphi}}\right)$ is the entire complex plane for all other permutations for every $\varepsilon \geqq 0$. Thus, from Theorem $3, S\left(\AA_{A^{(\varepsilon)}}\right)$ is determined by the sets $G^{\varphi_{i}}\left(\Omega_{\mathbf{A}_{(\varepsilon)} \varphi_{i}}\right)$, which turn out to be

$$
\begin{aligned}
G^{\varphi_{1}}\left(\Omega_{\boldsymbol{A}(\varepsilon)^{\varphi_{1}}}\right) & =\left\{\sigma: 1+2|\sigma-\varepsilon|-|\sigma-\varepsilon|^{3} \geqq 0\right\} \\
& =\{\sigma:|\sigma-\varepsilon| \leqq 1.62\},
\end{aligned}
$$

1 Here, we are describing permutations by their disjoint cycles. 


$$
\begin{aligned}
G^{\varphi_{2}}\left(\Omega_{A_{(\varepsilon)} \varphi_{2}}\right) & =\left\{\sigma: 1-2|\sigma-\varepsilon|-|\sigma-\varepsilon| \cdot|\sigma+\varepsilon|^{2} \geqq 0\right\}, \\
G^{\varphi_{3}}\left(\Omega_{\left.\left.\mathbf{A}_{(\varepsilon)}\right)_{3}\right)}\right) & =\left\{\sigma:-1+2|\sigma+\varepsilon|+|\sigma+\varepsilon|^{3} \geqq 0\right\} \\
& =\{\sigma:|\sigma+\varepsilon| \geqq 0.45\} .
\end{aligned}
$$

The basic reason for considering such an example is that, for suitable choices of $\varepsilon$, the actual number $r$ of permutations in Theorem 6 which are necessary to describe $S\left(\AA_{4(\varepsilon)}\right)$ can be made to vary from one to three. More precisely, for $0 \leqq \varepsilon<0.045, r=3$; for $0.045 \leqq \varepsilon<0.45$, $r=2$; and for $0.45 \leqq \varepsilon, r=1$. The first two cases are illustrated in Figures 1 and 2.

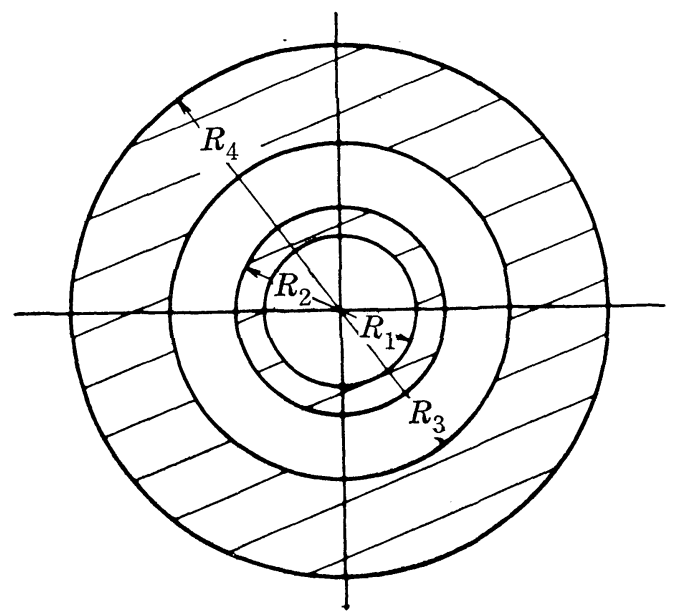

FIG. 1

$\varepsilon=0 ; R_{1}=0.45, R_{2}=0.62, R_{3}=1.00, R_{4}=1.62$

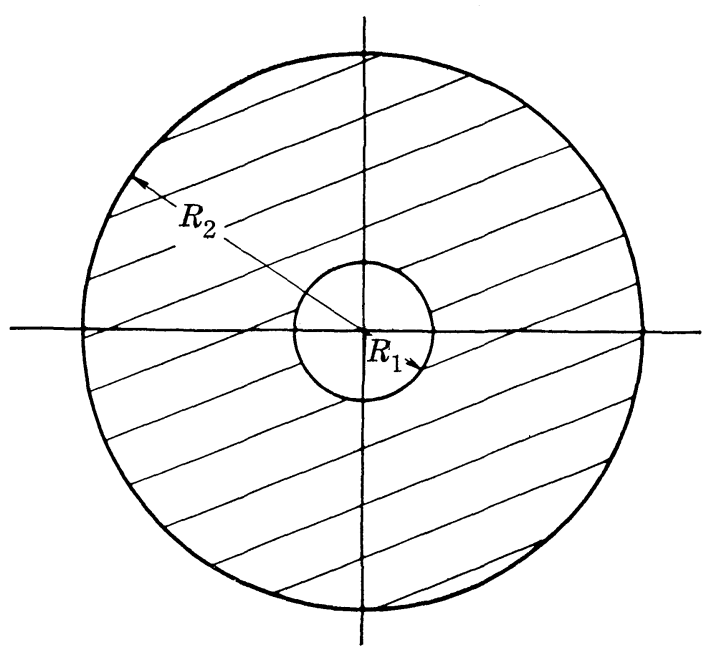

FIG. 2

$\varepsilon=0.05 ; R_{1}=0.40, R_{2}=1.67$ 
This last example serves to answer some questions which might naturally arise in reading the previous sections. First, it shows that $n \times n$ matrices $A$ exist for which at least $n$ permutations $\varphi$ are necessary to determine $S\left(\AA_{A}\right)$. On the other hand, it shows that it is not necessary for $A$ to be essentially diagonally dominant in order that $S\left(\Omega_{A}^{\circ}\right)$ coincide with $\operatorname{rot} G\left(\Omega_{A}\right)$ (cf. Theorem 1 ), since choosing $\varepsilon=0.5$ in (4.4) gives this condition. Finally, it demonstrates that, in general, it is not possible to find a single matrix $B \in \AA_{A}^{\circ}$ for which $S\left(\AA_{A}\right)$ is rot $S\left(\Omega_{B}\right)$. This fact follows quite easily from the last example with $\varepsilon=0.05$, in particular.

\section{BIBLIOGRAPHY}

1. Paul Camion and A. J. Hoffman, On the nonsingularity of complex matrices, Pacific J. Math. 17 (1966), 211-214.

2. H. G. Eggleston, Convexity, Cambridge at the University Press, 1958.

3. B. W. Levinger and R. S. Varga, Minimal Gerschgorin sets II, Pacific J. Math. 17 (1966), 199-210.

4. A. M. Ostrowski, Über die Determinanten mit Überwiegender Hauptdiagonale, Comment. Math. Helv. 10 (1937), 69-96.

5. Olga Taussky, On the variation of the characteristic roots of a finite matrix under various changes of its elements, Recent advances in matrix theory, edited by $\mathrm{H}$. Schneider, University of Wisconsion Press, 1964, 125-138.

6. Riehard S. Varga, Minimal Gerschgorin sets, Pacific J. Math. 15 (1965), 119-729.

7. - Matrix iterative analysis, Prentice-Hall, Inc. 1962.

Received October 29, 1964.

Case Institute of Technology

Cleveland, OHio 



\section{PACIFIC JOURNAL OF MATHEMATICS}

\section{EDITORS}

H. SAMELSON

Stanford University

Stanford, California

J. P. JANS

University of Washington

Seattle, Washington 98105

\section{J. DuGUNDJI}

University of Southern California

Los Angeles, California 90007

RICHARD ARENS

University of California

Los Angeles, California 90024

\section{ASSOCIATE EDITORS}

E. F. BECKENBACH
B. H. NEUMANN

F. WOLF

K. YoSIDA

\section{SUPPORTING INSTITUTIONS}

\author{
UNIVERSITY OF BRITISH COLUMBIA \\ CALIFORNIA INSTITUTE OF TECHNOLOGY \\ UNIVERSITY OF CALIFORNIA \\ MONTANA STATE UNIVERSITY \\ UNIVERSITY OF NEVADA \\ NEW MEXICO STATE UNIVERSITY \\ OREGON STATE UNIVERSITY \\ UNIVERSITY OF OREGON \\ OSAKA UNIVERSITY \\ UNIVERSITY OF SOUTHERN CALIFORNIA
}

\author{
STANFORD UNIVERSITY \\ UNIVERSITY OF TOKYO \\ UNIVERSITY OF UTAH \\ WASHINGTON STATE UNIVERSITY \\ UNIVERSITY OF WASHINGTON \\ AMERICAN MATHEMATICAL SOCIETY \\ CHEVRON RESEARCH CORPORATION \\ TRW SYSTEMS \\ NAVAL ORDNANCE TEST STATION
}

Mathematical papers intended for publication in the Pacific Journal of Mathematics should be typewritten (double spaced). The first paragraph or two must be capable of being used separately as a synopsis of the entire paper. It should not contain references to the bibliography. Manuscripts may be sent to any one of the four editors. All other communications to the editors should be addressed to the managing editor, Richard Arens at the University of California, Los Angeles, California 90024 .

50 reprints per author of each article are furnished free of charge; additional copies may be obtained at cost in multiples of 50 .

The Pacific Journal of Mathematics is published monthly. Effective with Volume 16 the price per volume (3 numbers) is $\$ 8.00$; single issues, $\$ 3.00$. Special price for current issues to individual faculty members of supporting institutions and to individual members of the American Mathematical Society: $\$ 4.00$ per volume; single issues $\$ 1.50$. Back numbers are available.

Subscriptions, orders for back numbers, and changes of address should be sent to Pacific Journal of Mathematics, 103 Highland Boulevard, Berkeley 8, California.

Printed at Kokusai Bunken Insatsusha (International Academic Printing Co., Ltd.), No. 6, 2-chome, Fujimi-cho, Chiyoda-ku, Tokyo, Japan.

\section{PUBLISHED BY PACIFIC JOURNAL OF MATHEMATICS, A NON-PROFIT CORPORATION}

The Supporting Institutions listed above contribute to the cost of publication of this Journal, but they are not owners or publishers and have no responsibility for its content or policies. 


\section{Pacific Journal of Mathematics

Vol. 19, No. $3 \quad$ July, 1966

S. J. Bernau, The spectral theorem for unbounded normal operators .......

Lu-san Chen, Asymptotic behavior of solutions of parabolic equations of

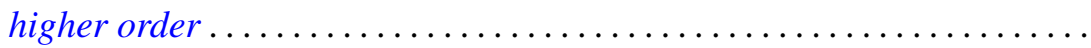

Lawrence William Conlon, An application of the Bott suspension map to the

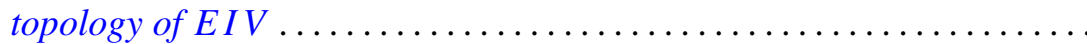

Neal Eugene Foland and John M. Marr, Sets with zero-dimensional kernels .........................................

Stanley Phillip Franklin and R. H. Sorgenfrey, Closed and image-closed

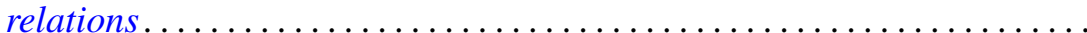

William Jesse Gray, A note on topological transformation groups with a

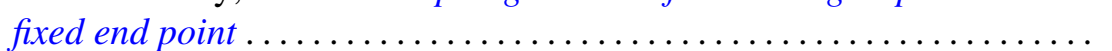

Myron Goldstein, $K$ - and L-kernels on an arbitrary Riemann surface ...... 449

George Joseph Kertz and Francis Regan, The exponential analogue of a generalized Weierstrass series .............................

Walter Leighton, On Liapunov functions with a single critical point ........ 467

Bernard Werner Levinger and Richard Steven Varga, On a problem of $O$.

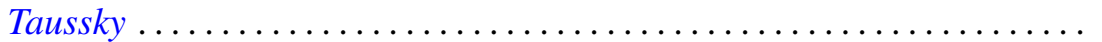

Lowell Duane Loveland, Tame subsets of spheres in $E^{3} \ldots \ldots \ldots \ldots \ldots . .489$

Erik Andrew Schreiner, Modular pairs in orthomodular lattices ......... 519

K. N. Srivastava, On dual series relations involving Laguerre polynomials ...............................

Arthur Steger, Diagonability of idempotent matrices.....

Walter Strauss, On continuity of functions with values in various Banach spaces...

Robert Vermes, On the zeros of a linear combination of polynomials ...

Elliot Carl Weinberg, On the scarcity of lattice-ordered matrix rings ....

Harold Widom, Toeplitz operators on $H_{p} \ldots \ldots \ldots \ldots \ldots$

Neal Zierler, On the lattice of closed subspaces of Hilbert space...

Irving Leonard Glicksberg, Correction to: "Maximal algebras and a theorem of Rado"

John Spurgeon Bradley, Correction to: "Adjoint quasi-differential operators of Euler type"

William Branham Jones, Erratum: "Duality and types of completeness in locally covex spaces".

Stanley P. Gudder, Erratum: "Uniqueness and existence properties of bounded observables" 